\title{
The needs and issues associated with representing and integrating multiple views of the enterprise
}

\author{
L. E. Whitman
}

Automation \& Robotics Research Institute

7900 Jack Newell Blvd. South

Fort Worth, TX 76118, U.S.A.

B. L. Huff

The University of Texas at Arlington

IMSE Dept. PO Box 19017

Arlington, TX 76019, U.S.A.

\author{
A. R. Presley \\ Truman State University \\ Division of Business and Accountancy \\ 100 East Normal \\ Kirksville, MO 63501, U.S.A.
}

\begin{abstract}
A critical component of any information infrastructure is a common understanding of the enterprise. Enterprise models enable this common understanding. The enterprise model can provide a comprehensive understanding of the environment the information infrastructure is designed to support. Models are typically developed from one of five perspectives or views. The different model views are presented and a comparison of these views is discussed. These five views are: business rule, activity, business process, resource, and organization views. The primary concern in this research is the identification of the issues of multiple views of an enterprise or system. Most project managers do not consider the issues pertaining to a multiple view model of a system. These managers develop and even
\end{abstract}


maintain multiple types of models for different purposes. These multiple types of models are generally developed on an ad hoc basis. By understanding the issues relating to maintaining multiple views of an enterprise, the benefits of multiple views can be realized while minimizing its difficulties. Three approaches to integrating multiple views are explained and their relative shortcomings are discussed.

\section{Keywords \\ Enterprise models, model views, extended enterprise, virtual enterprise}

\section{INTRODUCTION}

A model is a representation of reality used for analysis, design, and decision making. Models contain information necessary for the task at hand while omitting unnecessary details The typical uses of modeling are (Marca and McGowan 1988; Nathan and Wood 1991; Snodgrass 1993; Reimann and Sarkis 1996):

- To analyze and design the enterprise and its processes prior to implementation

- To help reduce complexity

- To communicate a common understanding of the system

- To gain stakeholder buy-in

- To act as a documentation tool for ISO 9000, TQM, Concurrent Engineering, and other efforts.

An enterprise may be represented in many different forms. Each of these representations provides a different view or perspective of the enterprise. These different views are required to support the design, analysis, and implementation of the enterprise. A primary thrust of this research is to understand the relationship between the various enterprise views. This paper discusses the needs and issues with representing and integrating multiple views of the enterprise.

\section{VIEWS}

Multiple perspectives of an enterprise are required due to the various questions and viewpoints of the end customers of an improvement effort. Previous research defines a number of different views. Computer Integrated Manufacturing Open Systems Architecture (CIMOSA) promotes four views: Function, Information, Resource, and Organization (Vernadat 1992). The Zachman Framework of 1987 (Zachman 1987) was extended by Sowa in 1992 (Sowa and Zachman 1992) and describes several different perspectives. Curtis (Curtis, Kellner et al. 1992), defines his four views as functional (what process elements are being performed, and what flows of information entities are relevant to these process elements), behavior (when process elements are performed (sequencing)), organizational or resource (where and by whom processes are performed, physical communications mechanisms, storage media and locations), and informational (what information entities are produced or manipulated by the process). The information entities include data, artifacts, products, and objects. ARIS (Architecture of Integrated 
Information Systems) also has four views. The three main views used are data, function, and organization. Depending on context (information or business system) the fourth view is either called the resource or control view (Scheer 1994). Previous work in the development of architectures by the Automation \& Robotics Research Institute (Presley, Huff et al. 1993) describes a five-view approach:

- Business Rule (or Information) View defines the entities managed by the enterprise and the rules governing their relationships and interactions,

- Activity View defines the functions performed by the enterprise (what is done),

- Business Process View defines a time-sequenced set of processes (how it is done),

- Resource View defines the resources and capabilities managed by the enterprise,

- Organization View describes how the enterprise is organized which includes the set of constraints and rules governing how it manages itself and its processes.

However, this does not mean that all these views must be present in all models. A model is an abstract representation of reality which should exclude details of the world which are not of interest to the modeler or the ultimate users of the model. Models are developed to answer specific questions about the enterprise. If the information modeled in a particular view is unnecessary to answer these questions, it may not be necessary to create the view. This research focuses specifically on the need for analysis of resource constraints and process flows. Most of the authors cited in the previous paragraphs, along with others have commented on the difficulty in relating the views to each other and maintaining consistency. This paper attempts to identify some issues of maintaining multiple views and provides some examples.

\section{THE NEEDS OF MULTIPLE VIEWS}

This section begins by describing a unique categorization of process types. This categorization is then used to describe the concepts underlying an extended enterprise. An additional concern regarding the use of models in general is then addressed as the concept of a living model of the enterprise is described.

\section{Categories of Processes}

Presley, et al., (Presley, Huff et al. 1993) propose that business processes may be placed into three categories: (1) those processes which transform external constraints into internal constraints (set direction), (2) those processes which acquire and make ready required resources, and (3) those processes which use resources to produce enterprise results. By providing categories to organize processes, more holistic enterprise designs may be achieved. Figure 1 shows activities (boxes) arranged into business processes (ellipses). The business 
processes are organized into an enterprise represented by the larger box. At this high level of abstraction, the enterprise itself is represented as an activity that takes inputs and transforms them into outputs using available resources under the bounds of a set of constraints.

Frequently the only activities or processes considered in modeling and improvement activities are those listed as category 3 which transform inputs into products and services. However, it is as important to consider the strategic and acquisition activities in an enterprise. Understanding the different process categories is vital to develop useful representations. Categorizing the different processes helps to ensure that the frequently overlooked categories of setting enterprise direction and acquiring and preparing resources are considered.

\section{Enterprise}

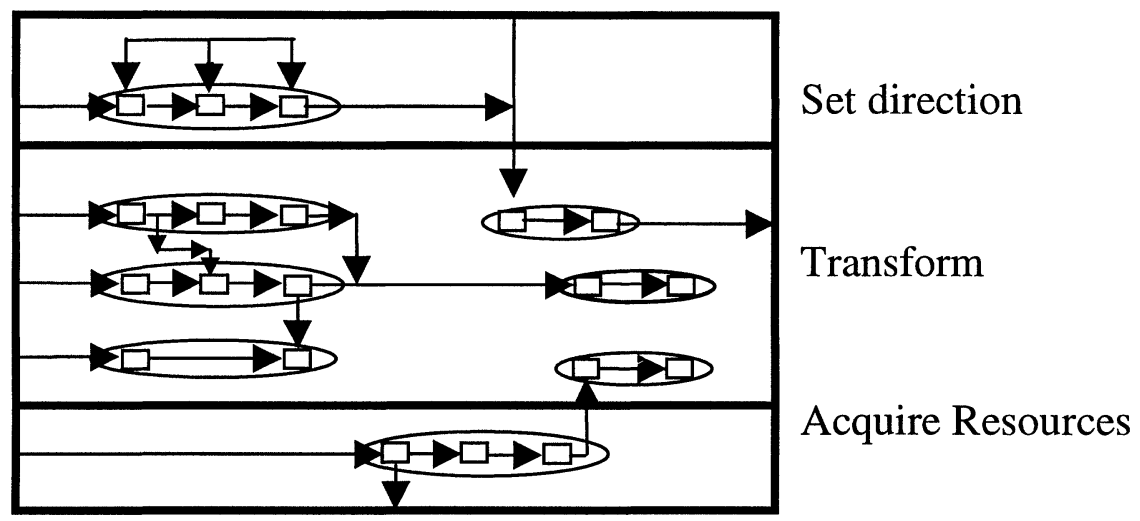

Figure 1: Process Categories

\section{Extended Enterprises}

The concept of a virtual or extended enterprise introduces several issues in the representation of an enterprise. An extended enterprise expands the scope of a model from bounding a single enterprise to include additional processes performed by other enterprises. Defining and coordinating all of the business processes comprising an extended enterprise is significantly more difficult than coordinating the actions of a single business entity. The increased interaction between the various enterprise processes adds to model complexity. The operation of a process oriented and highly flexible extended enterprise mandates that all activities, information, resources, and organizational issues be carefully integrated. Traditional process modeling and planning methods do not address the unique needs of inter-enterprise activities. This lack of support for the unique needs of the 
extended enterprise is in addition to the shortcomings discussed earlier. Figure 2 shows that the enterprise consists of a set of business processes from category (1) which are owned by each individual enterprise. The other enterprise models need not contain the details of category one processes. Category 2 and 3 processes relevant to the extended enterprise are included in all models. The formation of each extended enterprise should be viewed as a temporary or transient state. These enterprise structures should have the ability to form and dissolve based on dynamic market opportunities. This dynamic nature of the extended enterprise presents significant challenges to the modeling methods and tools designed to represent them. These extended enterprise modeling approaches must represent the core business processes of each business entity participating in the virtual organization. The model must also support the frequent addition, subtraction, and reconfiguration of models that represent the transient processes performed by the extended enterprise. The ability to support the frequent reconfiguration of the enterprise model to reflect the nature of an evolving enterprise requires the creation of a living model of the enterprise.

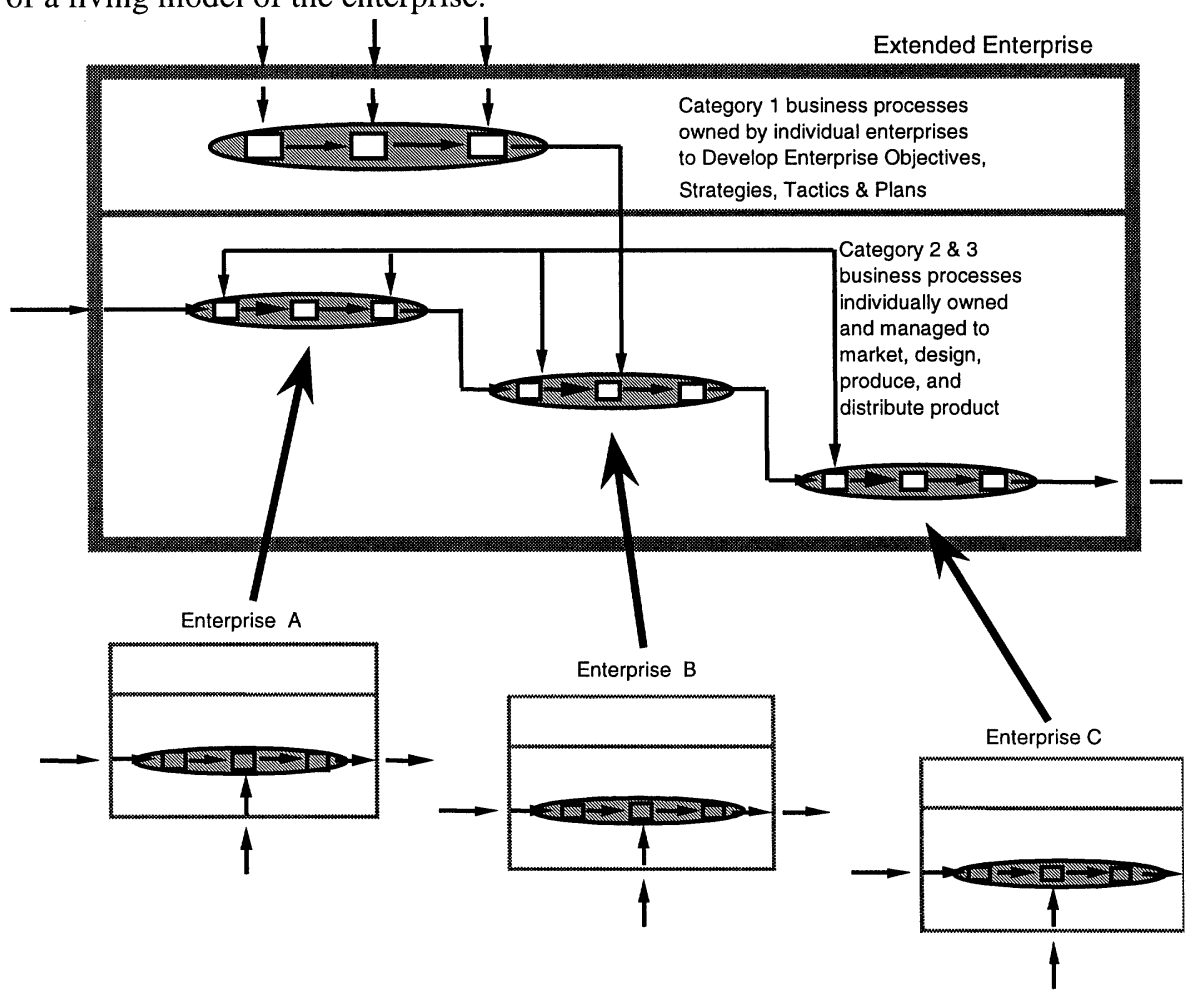

Figure 2: Extended Enterprise 


\section{A Living Model of the Enterprise}

An additional consideration to that of views or perspectives relates to the actual use of the model. The authors propose that the use, both future and present, of the model is vital to ensuring that the model serves the enterprise in the most effective manner. To this end, a living model of the enterprise is proposed. A living model of the enterprise is defined as a model that drives, and is driven by, the daily operations of the enterprise (Whitman and Huff 1997). These living models of the enterprise can be classified along three dimensions. Figure 3 shows these dimensions to be scope, enactment, and the dynamicness of the model. A description of each of these characteristics follows.

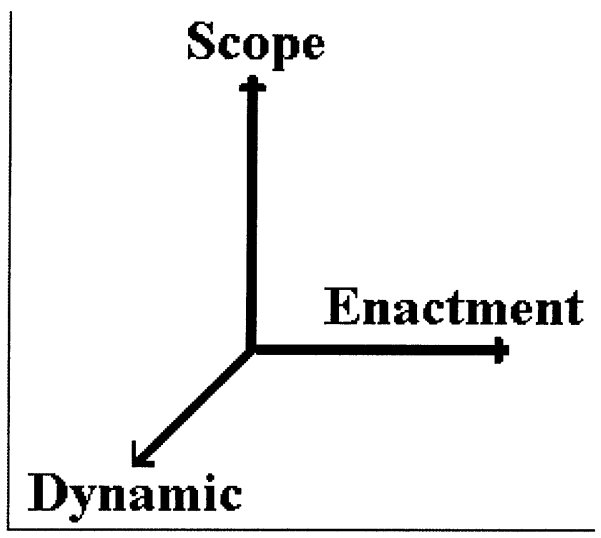

Figure 3: Living Model Dimensions

Scope is the pervasiveness of the model throughout the enterprise. Enterprise modeling by its very nature is intended to provide a holistic representation of the entire enterprise. It is sometimes necessary to bound the model to a subset of the enterprise. The bounds describe the scope of the model. Enactment is the level in which the model drives and is driven by the system. There is a wide variation in the enactment capabilities of a living model. A model can range from no enactment at all to driving the entire enterprise and providing all inputs and reporting the status of the enterprise when requested. Some more likely phases of enactment might be to use a work flow arrangement which can provide either direction to enterprise personnel allowing them to deviate slightly from the process or require strict adherence to the process. Dynamic is the ability to respond to both permanent and temporary process changes to the system. As has been previously discussed, an important living characteristic of an enterprise model is its ability to change. This dimension denotes this ability. Most models today do not facilitate the ease of change for the model. The phases of dynamic range from no capability to the model itself being capable of learning from its environment and then modifying 
itself to reflect and implement the new process. This dynamic dimension is not to be confused with simulation models, which are often called dynamic representations.

\section{ISSUES BETWEEN VIEWS}

In this section we discuss four key issues in the synthesis between views. These issues are: 1) gaps in the view, 2) differences in methodology structure, 3) artificial wrappers (decomposition versus aggregation), and 4) model ambiguities.

\section{Gaps in the view}

The primary reason for modeling is to answer a question. Details that are important to the question answered by the model are included. Therefore, some information that is pertinent in one view may not be relevant in another view. This is the advantage of modeling multiple views of an enterprise. While there is significant overlap between the activity, process, and organization views, there are also significant portions of each view that are not described in the other views. All information about a given system or enterprise can not be represented in a single view. Complex questions regarding the enterprise often require analysis across multiple views of that system. While the various modeling methods are capable of representing each of these enterprise views independently, they do not directly support the integration of their view with the other modeled enterprise views. This lack of view integration inhibits the development of a holistic understanding of all the diverse, yet interrelated, concepts and issues which can impact the performance and behavior of the modeled system.

\section{Artificial Wrappers}

Models are frequently created with a hierarchical structure that provides a useful tool for understanding an enterprise or system. Hierarchical modeling utilizes a subordinate principle of abstraction called decomposition (Rumbaugh, Blaha et al. 1991), which is the breaking down of each activity into more detail in a continuous manner until the greatest level of detail is achieved (Marca and McGowan 1988). An example of this top down modeling strategy, as used in the IDEF0 method, is shown in figure 4. 


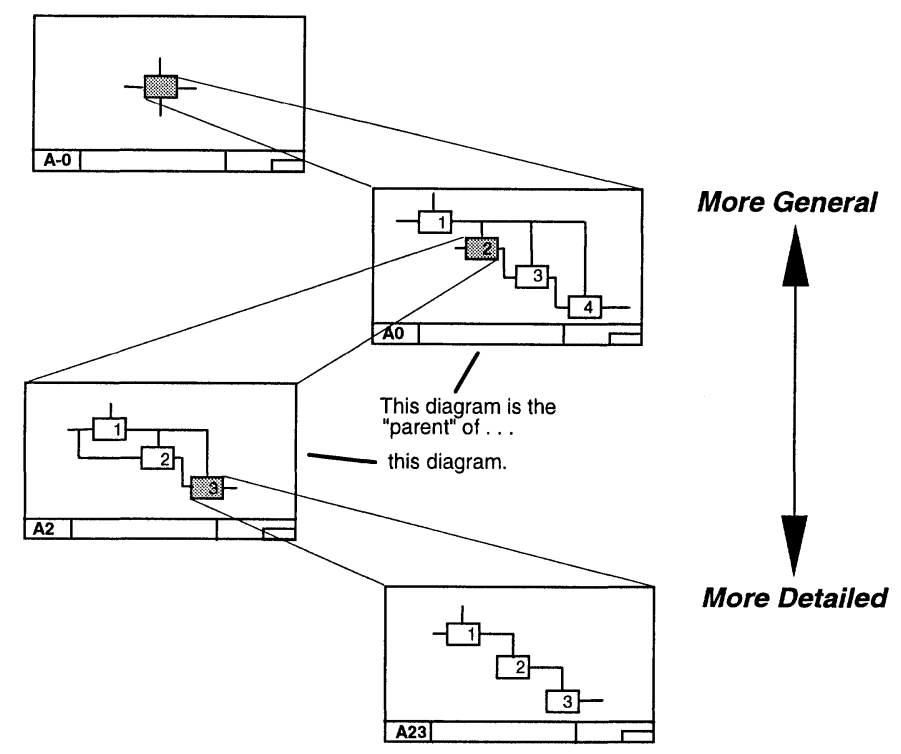

Figure 4: Functional Decomposition

While the use of hierarchical modeling strategies are beneficial due to their ability to hide system complexity, the modeling procedures dictated by the methods often force the definition of abstracted entities which are not readily recognized by individuals familiar with the system. Most of the reviewers of models are familiar with either the top levels of the system or with the bottom levels of the system. Generally familiar "labels" for these levels are used that provide a frame of reference for these reviewers. In the previous figure, the A-0 and A0 level would likely use similar terms for the activities identified at these levels. Also, the A23 level would also use familiar terms for the activities at lower levels. However, the middle level activities, such as those in A2, might not be recognized by anyone familiar with the system. Therefore, to properly decompose these activities, an "artificial wrapper" is placed on groupings to aggregate the lower level activities to match these with the higher level activities. Most systems are well defined at the high levels and at the low levels, but are not explicitly defined at the middle levels. In the author's experience, this has confused many model reviewers.

\section{Differences in Structure}

The structures of the various views are not necessarily conducive to mapping between views. Activity and process views facilitate a hierarchical representation, whereas, the data view does not. A high level activity view may contain 
"documents" as a data element, and a lower level activity view contains "work authorization." The data view would contain only the element "work authorization" with the data required on the fields of the form. A mapping technique might be to only map the lowest level of the hierarchical method, but this approach loses the advantages of a hierarchical model in reducing the complexity of the system and increasing the understanding of the system.

\section{Model Ambiguities}

Information that is pertinent in one view is frequently not included in another view. This reduction of information is useful for a clearer understanding of the system. However, this also leads to ambiguities in the model. Some concepts are easy to represent in one view of the model, but in the complementary view, additional information is required to remove ambiguities. Additionally, an alternate representation of the same concept in different views often leads to ambiguity. Part of the ambiguity arises from the very nature of the views and their focus. For example, the process view by necessity focuses on the transformations that objects and information in the enterprise go through. While the objects and information transformed are usually shown in the view (although some modeling methods may not explicitly identify them), the focus is still on the transformation. On the other hand, these same objects and information would be the primary focus of the business rule or information view. The transformations that might have bound the objects together in the activity view may be hidden or not shown at all in the information view. Also, some views enforce artificial decompositions on objects. This is the case in some information view methods, which break apart real world objects into abstract concepts to support the needs of the view. They may also create new concepts, which do not actually exist in the real world, again to facilitate the representation of the view. So a user wanting to analyze a concept in one view might have difficulty identifying the concept in another view or may find that the concept has been divided into several other concepts in the other view. While object oriented approaches help mitigate some of these ambiguities, these inter-view ambiguities still exist.

In addition to inter-view ambiguities, reduction of information may lead to intraview ambiguities. We clarify this point with the following example. In figure 5, an activity is shown with two inputs. This is a correct representation of the process, but it is ambiguous. The two inputs represent one of three conditions. An assemble, a match, or a selection. An assemble means the two inputs could represent two items needed for the activity together. An example could be a motherboard and a cpu. Any one motherboard may be assembled with any cpu (assuming this is a line with only one cpu and one type of motherboard). A match means that a specific instance of Input 1 must be matched with Input2 for the activity to occur. An example could be a part to be machined and its part program. A selection means that only one of the two inputs are needed for the activity to occur. An example of this can be seen in figure 6. This example shows where the part is being 
manufactured and if not correct cycles back to the manufacturing process. Therefore, either type of input is required for the activity to occur.

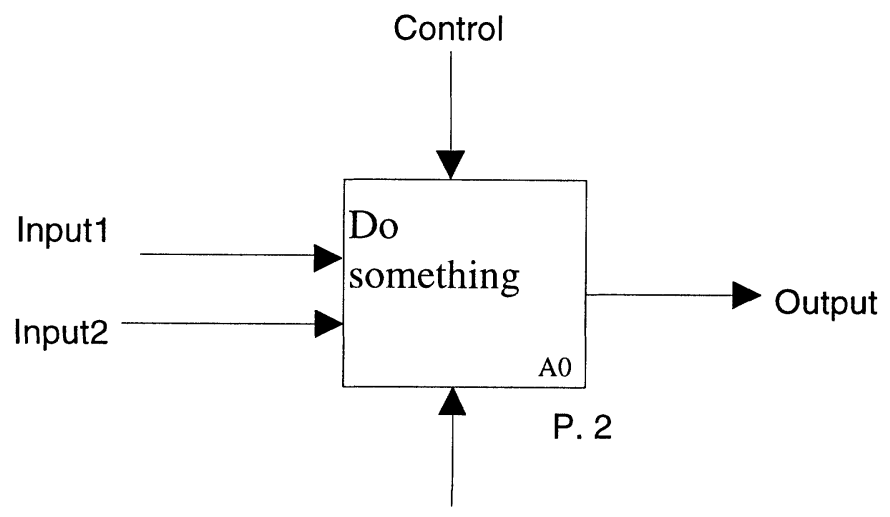

Mechanism

Figure 5: Example of ambiguity

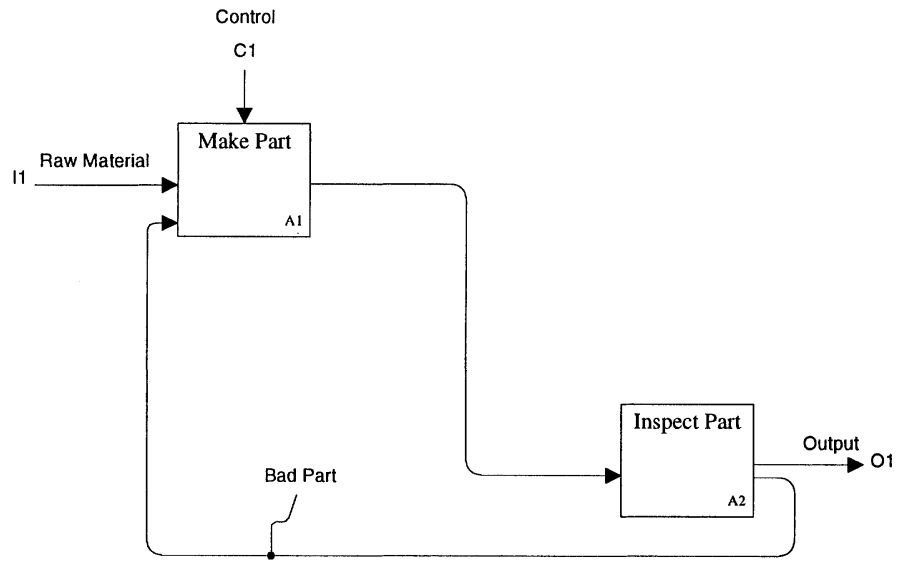

Figure 6: Selection Activity

\section{CONCLUSION/FUTURE RESEARCH}

A comprehensive understanding of the need and issues related to multiple views of an enterprise can aid in the implementation of intelligent manufacturing systems. This paper discussed the five perspectives typically used in most modeling efforts. Most analysis efforts develop and maintain multiple types of models for different purposes. Resolution to the issues identified in this research related to the 
inconsistencies encountered in modeling are critical to improving the design and analysis of any system. By understanding the issues relating to maintaining multiple views of an enterprise, the benefits of multiple views can be realized while minimizing the resultant difficulties with the consistency of multiple views. Some methods reduce complexity to aid in achieving understanding. However, this is not without cost. The reduction of complexity is achieved by the reduction of details. These details are critical for certain purposes such as temporality. Multiple views enable the reduction of complexity in one view and allow the vital details to be described in another view.

Presley (1997), provides an IDEF0 model describing his methodology. He integrates the views through the IDEF5 Ontology Capture Method. An IDEF0 model describing the methodology is shown in figure 7. The IDEF5 model serves as the business rule view. The necessary relationships are extracted from the IDEF5 model and are used to specify the organization and resource views. Activity information from the IDEF5 model is used to generate an IDEF0 activity view and IDEF3 business process view models. 


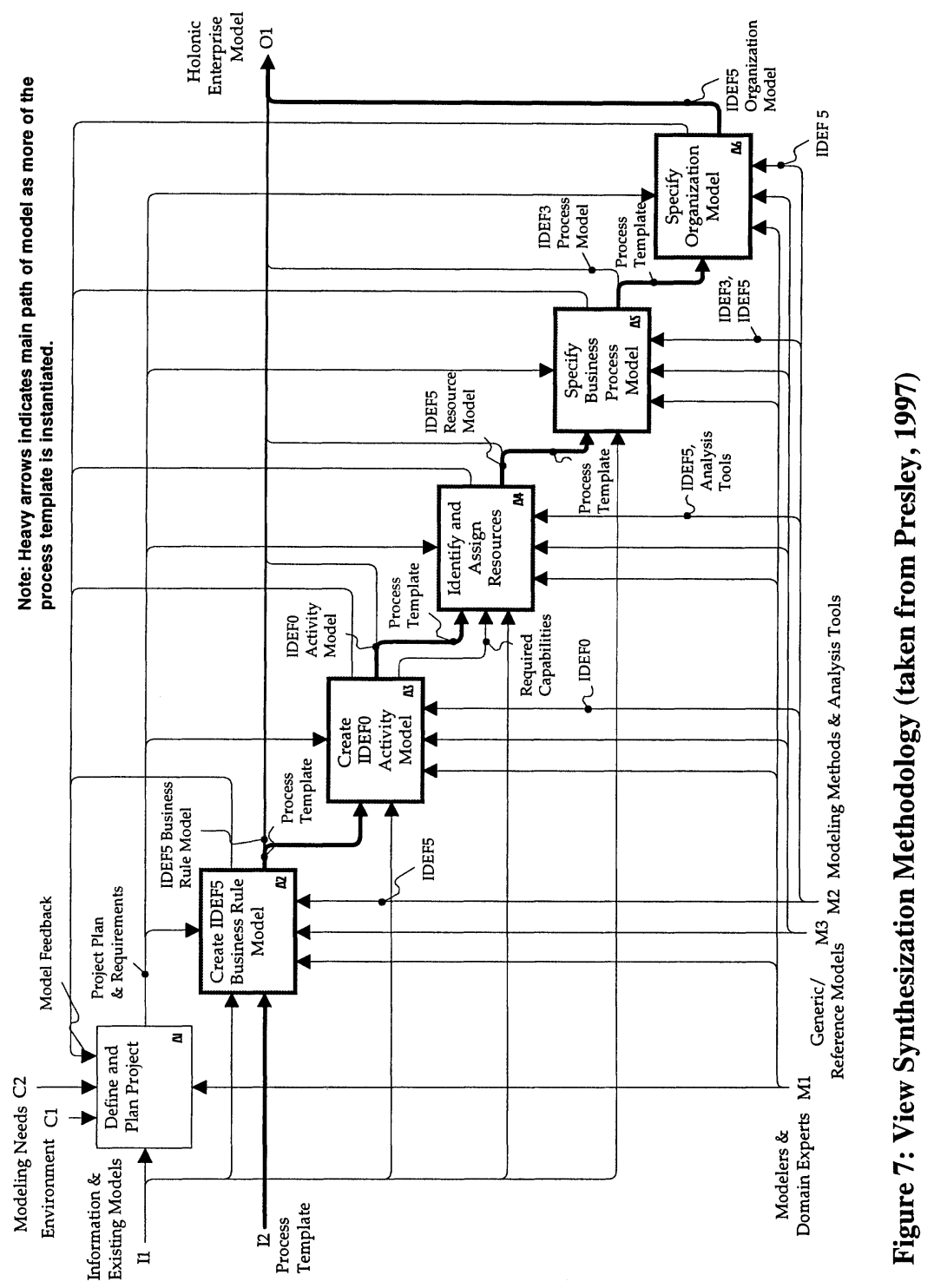


The resolution of these model view integration issues is becoming increasingly more important as the complexity of our manufacturing enterprises continue to increase. The lack of simple model integration strategies will hinder the development of living models of the enterprise. Our research will continue to explore the courses of poor model view integration as we undertake the next generation of enterprise model strategies.

\section{REFERENCES}

Curtis, B., M. I. Kellner, et al. (1992). "Process Modeling." Communications of the ACM 35(9): 75-90.

Marca, D. A. and C. L. McGowan (1988). SADT: Structured Analysis and Design Technique. New York, NY, McGraw-Hill Book Co., Inc.

Miller, J. (1978). Living Systems. New York, McGraw-Hill Book Company.

Nathan, B. and J. Wood (1991). The use of IDEF0 to Document a methodology - a Novices Point of View, Automation \& Robotics Research Institute, Fort Worth, Texas.

Presley, A., B. Huff, et al. (1993). A Comprehensive Enterprise Model for Small Manufacturers. 2nd Industrial Engineering Research Conference, Los Angeles, CA.

Presley, A. R. (1997). A Representation Method to Support Enterprise Engineering. Industrial and Manufacturing Systems Engineering. Arlington, University of Texas at Arlington.

Reimann, M. D. and J. Sarkis (1996). "An integrated functional representation of concurrent engineering." Production Planning and Control 7(5): 452-461.

Rumbaugh, J., M. Blaha, et al. (1991). Object-Oriented Modeling and Design. Englewood Cliffs, NJ, Prentice-Hall.

Scheer, A.-W. (1994). Business Process Engineering: Reference Models for Industrial Enterprises. Berlin, Springer-Verlag.

Snodgrass, B. N. (1993). Integrating Activity Based Costing with IDEF Modeling. Dallas.

Sowa, J. F. and J. A. Zachman (1992). "Extending and Formalizing the Framework for Information Systems Architecture.” IBM Systems Journal 31(3): 590-616.

Vernadat, F. (1992). CIMOSA - A European Development for Enterprise Integration Part 2 Enterprise Modelling. Enterprise Integration Modeling: Proceedings of the First International Conference, Austin, TX, The MIT Press.

Whitman, L. E. and B. L. Huff (1997). A Living Enterprise Model. The Sixth Industrial Engineering Research Conference, Miami Beach, FL.

Zachman, J. A. (1987). "A Framework for Information Systems Architecture." IBM Systems Journal 26(3): 276-292.

LARRY WHITMAN is a Research Engineer with the Automation \& Robotics Research Institute (ARRI) of The University of Texas at Arlington (UTA). He is currently pursuing his Ph.D. degree from the Industrial and Manufacturing 
Systems Engineering department at UTA. He received his MSIE and BSET degrees from Oklahoma State University. Prior to joining ARRI, he spent ten years in the aerospace industry integrating factory automation and developing and supporting CAD systems. His research interests are in enterprise modeling, simulation, strategic cost justification, and enterprise applications in manufacturing.

BRIAN HUFF is an Assistant Professor in the Industrial and Manufacturing Systems Engineering Department at the University of Texas at Arlington (UTA). He received his Ph.D. and M.S. degrees from UTA. He received his B.S. in Petroleum Engineering from West Virginia University. His research interests are in manufacturing systems design, industrial simulation, industrial automation and robotics, and shop floor production execution and control.

ADRIEN PRESLEY is an Assistant Professor in the Division of Business and Accountancy at Truman State University. Previously he was a Research Industrial Engineer with the Automation \& Robotics Research Institute (ARRI) of The University of Texas at Arlington (UTA). He received his Ph.D. degree from the Industrial and Manufacturing Systems Engineering department at UTA. His research interests include enterprise modeling and analysis and management of technology. 